\title{
Summary of laser plasma physics sessions at the first AAPPS-DPP conference
}

\section{Zheng-Ming Sheng ${ }^{1,2,3}$}

Received: 26 July 2018 / Accepted: 28 September 2018 / Published online: 15 October 2018

(c) The Author(s) 2018

\begin{abstract}
The presentations on laser plasma physics at the first AAPPS-DPP conference covered topics of inertial confined fusion physics and technologies, laboratory astrophysics and high-energy density physics, laser plasma-based particle acceleration (electrons and ions) and radiation, fundamental laser plasma physics, and related high-power laser system development. This report summarizes the major advances in these topics presented at the plenary and oral sessions.
\end{abstract}

Keywords Laser plasma - Inertial confined fusion · High-energy density physics · Plasma-based particle acceleration $\cdot$ High-power laser

\section{Introduction}

The program for laser plasma physics includes five plenary talks and eight oral sessions (see Abstracts of the first Asia-Pacific Conference on Plasma Physics in the reference links). The five plenary speakers come from China, Japan, Korea, and France. One of these plenary talks on laser plasma-based laboratory astrophysics was selected by the solar and astrophysics program committee. Among the oral sessions, two sessions were organized jointly with the Asian Committee on UltraIntense Laser (ACUIL), the Asian collaboration network for high-intensity lasers. There were a total of 27 invited talks and six oral talks presented in the oral sessions and four posters in a poster session. Among the invited and oral presentations, seven talks were on inertial confined fusion, 12 talks on laser-driven particle acceleration and radiation, ten talks on high-energy density physics driven by lasers, and four

Zheng-Ming Sheng

zmsheng@sjtu.edu.cn; z.sheng@strath.ac.uk

1 Laboratory for Laser Plasma, School of Physics and Astronomy, Shanghai Jiao Tong University, Shanghai 200240, China

2 SUPA and Department of Physics, University of Strathclyde, Glasgow G4 0NG, UK

3 Tsung-Dao Lee Institute, Shanghai Jiao Tong University, Shanghai 200240, China 
talks on high-power laser systems and technologies. There were five invited and oral presentations from France and UK. In this summary report, I try to highlight the main reported progresses in the four different topics with the help of many speakers. A concluding remark is made at the end of the summary.

\section{Inertial confined fusion driven by lasers}

The investigation on inertial confined fusion (ICF) remains one of main interests in laser plasma and is one of the main drive forces for the development of this area ever since 1970s (Kaw 2017). Even though tremendous new progresses have been made in the central hotspot ignition scheme for ICF driven by the indirect-drive (ID) approach and direct-drive (DD) approach (Hurricane et al. 2014; Campbell et al. 2017), there are still significant challenges regarding fusion ignition and fusion energy (Edwards and Danson 2015). Experiments on NIF in LLNL suggest that both peak ablation pressure and the implosion velocity need to be further enhanced, and the hydrodynamic instabilities need to be further controlled to improve the implosion symmetry. New ignition schemes for ICF are still highly desired to push the ICF research forward.

The plenary talk by He [P1] reported a novel hotspot ignition scheme for ICF driven by a so-called hybrid-drive (HD) approach (He et al. 2016). This is, however, not just simply combining the laser ID and DD approaches. This scheme runs in two phases. In the first phase, a layered fusion fuel capsule inside a spherical hohlraum is ablated by lower ID radiation temperature, resulting in the pre-compression of fusion fuel and the formation of a long-scale corona plasma. In the second phase, the DD lasers are injected and absorbed near the critical surface, generating a supersonic electronic-thermal wave (ETW). The ETW is significantly smoothed during propagation in the long-scale ID corona plasma and finally slows down to a sonic ETW. This process behaves like a "snow plow", piling up the low corona plasma density into the high-density platform between the sonic wave front and the ID ablation front, and resulting in a far higher HD plasma pressure than the ID ablation pressure. The resulting HD pressure not only strongly suppresses the hotspot deformation and the hydrodynamic instabilities, but also provides a large $\mathrm{PdV}$ work on the hotspot and results in non-stagnation ignition according to their simulation. The experiments on the boosted HD pressure and smoothing effect of supersonic ETW are being carried out at the ShenGuang (SG)-III laser facility (Zheng et al. 2016, 2017).

In his plenary talk, Miquel [P13] presented an overview on the Laser Megajoule (LMJ) program in the French Alternative Energies and Atomic Energy Commission (CEA). The French Fusion program combines the physics models, numerical simulation and experimental validation (Miquel 2016). Six experimental configurations have been defined during the ramp-up of LMJ. LMJ has been working in the second configuration ( 2 bundles $=16$ beams, $60 \mathrm{~kJ}, 4$ diagnostics) since the end of 2016, providing good overall performances. Three other bundles are mounted and will be activated the next year (third configuration, $150 \mathrm{~kJ}, 10$ diagnostics). Three activities are performed at the same time: mounting of new 
bundles, commissioning of the previous assembled bundles, and physics experiments. CEA is developing a thematic approach on LMJ and has defined eight experimental topics for the simulation program. Several physics campaigns have been performed since 2014 and have addressed three of these topics. About ten experiments are planned till 2019 and will address six different topics. The first implosions with D2 + Ar capsules are planned in 2019. In addition, associated with the LMJ, the PETAL laser has been developed (Batani et al. 2014; Casner et al. 2015). This is a multi-PW beam coupled to LMJ, which offers the opportunity to study a wider area of physics. A record of $1.2 \mathrm{PW}$ (840 J-700 fs) was obtained in 2015. Pulse duration was improved (570 fs) and should bring the power to 1.8 PW. For academic access to LMJ-PETAL, two calls of proposals for 2017-2020 have received a great success (25 proposals), and six experiments have been selected by the international scientific advisory committee. LMJPETAL was ready for the first international academic experiments in December 2017.

To achieve ICF fusion ignition, it is required that a hohlraum should produce a very high symmetric X-ray drive in the ID approach (Lindl 1995; Atzeni and Meyerter-Vehn 2004), which remains a major challenge for ICF. In the invited talk by Lan [L-I21], a novel spherical hohlraum with six cylindrical laser entrance holes (LEHs) of octahedral symmetry was presented, called as the octahedral spherical hohlraum (Lan et al. 2014a, b, c). It is shown that such a hohlraum can not only provide the capsule a radiation drive with natural, robust and very high symmetry without any supplementary technology, but also a high-energy coupling efficiency from the hohlraum to the ignition hotspot. Since 2013, considerable progresses have been made in the design of the octahedral spherical hohlraum (Huo et al. 2014; Li et al. 2015; Shu et al. 2015; Duan et al. 2015; Cao et al. 2017; Lan et al. 2016). Meanwhile, a series of experiments of the Spherical Hohlraum Campaign (SPHC) have been performed on the ShenGuang laser facilities in Shanghai and Mianyang in China to test its performance (Huo et al. 2016a, b; Xie et al. 2016; Chen et al. 2017; Li et al. 2017c; Shu et al. 2018). Finally, it is proposed to use $2 \omega$ lasers for future ignition facilities with a configuration designed for the octahedral spherical hohlraum and to use a foam wall to increase the coupling efficiency from the laser to the capsule (Lan and Song 2017; Chen et al. 2018).

In indirect-drive ICF, the symmetric radiation drive is critical to the capsule implosion but difficult to achieve due to the non-uniform laser radiation inside the hohlraum. Nevertheless, the angular distribution of the radiation flux escaping from the hohlraum LEH is sensitive to non-uniform radiation properties such as laser spot and plasma motion, which therefore provides an effective way to study the hohlraum radiation properties. The invited talk by Yang [L-I24] reported an experimental investigation on the hohlraum radiation properties through the angular distribution of the radiation temperature performed on the SG-III prototype in Mianyang. Comparison between the experimental observations and the two-dimensional radiationhydrodynamic simulations was carried out (Zhang et al. 2014, 2016a, b; Hao et al. 2014; Yang et al. 2014). The difference between the 2D simulations and the real 3D effects caused by limited beam numbers on SG-III prototype laser facility was considered, and the influence of power balance and pointing accuracy of laser beams 
on the measured results was evaluated. Both vacuum- and gas-filled hohlraums were used to study the non-uniform radiation properties by investigating the angular distribution of the X-ray flux.

In addition to the conventional ICF schemes of central ignition, there were some talks on advanced ignition schemes, for example, the fast ignition (FI) scheme (Kodama et al. 2001). Gu [L-I16] reported their experimental and theoretical progresses on fast ignition (FI). By use of SG-II upgraded (SG-IIU) laser facility in Shanghai, delivering totally $24 \mathrm{~kJ}$ nanosecond laser ( 8 beams) for compression and $1 \mathrm{~kJ}$ at $10 \mathrm{ps}$ heating laser, several decomposed and integrated FI experiments were performed recently ( $\mathrm{Gu}$ et al. 2015). Pre-compression experiment was carried out to obtain the density and area density of the cone-in-shell FI target driven by the indirective approach. Some flat-target experiments were used to test the capability of the picosecond laser. Based on these decomposed experiments, the integrated experiments via the cone scheme were performed and a 200 -fold neutron enhancement was observed. The results confirmed the favorable heating effect of the picosecond lasers, where the heating efficiency can reach about $10 \%$ calculated from the measurements of neutron yields, density and spot size. Following these encouraging results, they have planned to carry out more FI experiments with their scheme in the future.

In recent years, it is found that high magnetic fields may significantly improve the performance of ICF both for conventional schemes and fast ignition schemes. For fast ignition, electron guiding by external magnetic fields in dense plasma was demonstrated by experiments, as presented in the talk by Santos [L-I21]. Using highenergy ns pulses, they observed that a single-turn coil produced discharge currents of $100 \mathrm{kA}$ and then magnetic fields $\sim 500 \mathrm{~T}$ were generated, as measured by proton deflectometry and high-frequency bandwidth B-dot probes (Santos et al. 2017, 2015; Law et al. 2016; Morace et al. 2017). Hot electrons ejected from the irradiated cathode provide the source for the quasi-static super-Alvénic current (Tikhonchuk et al. 2017). The magnetic field was successfully applied in experiments of lasergenerated relativistic electron transport into solid matter. The imposed magnetic field of $\sim 600 \mathrm{~T}$ yielded an unprecedented enhancement of a factor five on the relativistic electrons energy-density flux at $60 \mu \mathrm{m}$ depth as compared to unmagnetized transport conditions. The transported electron beams were characterized by imaging the coherent transition radiation emitted from the targets' rear surface in conjunction to benchmarked 3D PIC hybrid simulations, which were coupled to a coherent transition radiation post-processor. Under optimized experimental parameters, $70 \%$ of the electron beam energy is transported to the rear side within the size of the electron beam source (Bailly-Grandvaux et al. 2018).

Based upon the numerical simulation, Wang [L-I25] presented a magnetically assisted (MA) scheme for fast ignition. He and his collaborators developed an integrated simulation approach (Wang et al. 2015a), the so-called "two-system PIC" model, in which the fast electron generation by laser-plasma interactions and electron transport in real-density plasma (up to $300 \mathrm{~g} \mathrm{~cm}^{-3}$ ) can be simulated integratedly. In this way, the energy coupling from the laser to the target core region can be calculatedly self-consistently. In their simulations, they changed the density scale length of preplasmas, corresponding to different laser contrasts and found that the 
preplasmas in the cone had significant effects on laser-to-core coupling (Wang et al. 2015b), which may explain the discrepancy found in different experiments with the cone scheme (Key et al. 2008; Theobald et al. 2011; Shiraga et al. 2011). To reduce the preplasma effect, they proposed the MA fast ignition scheme using a cone-free target supplemented by an external 20-megagauss magnetic field to guide the fast electron motion (Wang et al. 2015b). It was found that laser-to-core coupling with this scheme can reach $14 \%$, which is much higher than that without the magnetic field and that with the cone scheme, typically at around 2 and $6 \%$, respectively. More recent simulations suggest that fusion ignition conditions may be reached with heating lasers at the $30 \mathrm{~kJ}$ level (Wang et al. 2016).

Some progress in block ignition scheme was presented by Wang [L-I3]. Fusion reactions of protons with the boron isotope 11 (HB11) can be considerably enhanced when PW laser pulses in picosecond duration are used to ignite fusion in a nonthermal way by direct conversion of laser energy into acceleration of plasma blocks, which have charge neutrality (Hora et al. 2015). This block acceleration was discovered before (Sauerbrey et al. 1996), which may be used to solve the difficulties of the HB11 reaction (Hora et al. 2010). Preliminary experimental results were reported by some European groups (Picciotto et al. 2014). A further improvement is due to measurements of an avalanche reaction (Hora et al. 2015, 2016) that was explained by elastic nuclear collisions in non-equilibrium plasmas (Eliezer et al. 2016). Furthermore, properties of the plasma explosion process were studied recently by Wang's group with multi-fluid hydrodynamics and PIC simulation (Li et al. 2017a; $\mathrm{Xu}$ et al. 2016).

Shigemori [L-I23] presented a talk on a new type of ablators for direct-drive ICF targets. Both the neutron yield and areal density for ICF ignition depend significantly on the level of laser imprinting (Nora et al. 2014). To mitigate the laser imprinting, investigations were carried out previously by thermal smoothing (Obenschain et al. 2002; Karasik et al. 2015; Nishikino et al. 2002). However, thermal smoothing is not effective either for long wavelength perturbations or when the effective separation distance between laser absorption region and ablation front is small in very early timing. Recently, they performed experimental investigation on the effects of diamond strength on surface perturbation due to the irradiation nonuniformity. In experiments, the target foils were irradiated with a foot pulse at the intensity of $4.0 \times 10^{12} \mathrm{~W} / \mathrm{cm}^{2}$ (low foot) or $5.0 \times 10^{13} \mathrm{~W} / \mathrm{cm}^{2}$ (high foot). The foils were subsequently accelerated by uniform main pulses of $\sim 10^{14} \mathrm{~W} / \mathrm{cm}^{2}$. For irradiation non-uniformity $\sim 10 \%$, the experimental results show that the imprinting amplitude (fundamental) was reduced for the diamond foil due to the material compressibility, in good agreement with $2 \mathrm{D}$ hydrodynamic simulation results.

\section{Laser-driven particle acceleration and radiation in plasma}

Laser-driven particle acceleration and radiation in plasma have been topics of significant interest in the last two decades with the advancement of high-power laser technology. In the recent decades, because of significant investment in Asian countries, 
in particular, in China, Japan, Korea, and India, both laser facilities and research outputs are at high levels. These are partially reflected in the presentations at this conference.

\subsection{Laser plasma-based electron acceleration}

Laser wakefield acceleration (LWFA) of electrons has a great potential for future compact accelerators due to its extremely high acceleration gradients (Tajima and Dawson 1979). Significant progress has been made in the last two decades in this area, which was built upon the new understanding of the related physics and the technical development of ultrashort high-power lasers and plasma targets. Currently, major efforts are being made for the improvement of the electron beam quality (low energy spread, small emittance, high stability) (Esarey et al. 2009), enhancement of beam energy (Wang et al. 2013; Kim et al. 2013; Leemans et al. 2014), and the development of various radiation sources from the accelerated electrons for applications (Giulietti 2016).

With multi-PW laser pulses, LWFA has the possibility to produce over $10-\mathrm{GeV}$ electron beams in a single stage with an acceleration distance of a few tens of centimeters. With conventional linear accelerators, it requires kilometer scale of acceleration distance to obtain the same energy level. In the plenary talk, Kim [P 7] presented an overview of LWFA and relevant activities at the Center for Relativistic Laser Science (CoReLS) in Gwangju, Korea. Multi-GeV beams have been demonstrated in the last few years (Kim et al. 2013, 2017). Recently, one of the two PW beamlines has been upgraded to a 4-PW 20-fs laser to explore relativistic laser-plasma interactions and strong field QED effects (Sung et al. 2017). The interaction chamber for LWFA was upgraded to accommodate the 4-PW laser pulses and performed commissioning experiments in 2017. In the commissioning experiments, electron beams at $\sim 4.5 \mathrm{GeV}$ with an energy spread about $20 \%$ was obtained by focusing a positively chirped 30-fs 52-J laser pulse by F/50 spherical mirror on a $7 \mathrm{~cm}$ gas medium at the density of $1.5 \times 10^{18} / \mathrm{cm}^{3}$. The gas medium was designed to be length variable with uniform gas density by combining the advantages of slit nozzle and gas cell, named as "Slitcell" (Aniculaesei et al. 2018). The mixed gas will be used to test ionization injection for reduced beam energy spread and higher electron energy over $10 \mathrm{GeV}$, which can be applied to generate bright gamma rays by Compton backscattering as well as to investigate the strong field QED effects via the laser electron collision.

In the plenary talk, Hosokai [P21] reported the status of the LWFA research project under the ImPACT-UPL Program in Japan. This 5-year program starting from 2014 was funded by Japanese government for promoting innovative and high-impact $\mathrm{R}$ and Ds. As one of the projects of this program, Project 1 on LWFA and XFEL aims to realize repeatable $\mathrm{GeV}$ class accelerator with the staging LWFA scheme. With electron energy gain over $1 \mathrm{GeV}$ in $10 \mathrm{~cm}$, energy spread less than $1 \%$, beam divergence less than $1 \mathrm{~mm}$ mrad, the beam could be used to generate $1 \mathrm{keV} \mathrm{X}$-ray beam by a microundulator with a length of $10 \mathrm{~cm}$ or less. Plasma micro-optics (PMO) as one of key techniques for stable beam generation provide excellent 
stability of electron beams (Mizuta et al. 2012; Nakanii et al. 2015), which can be produced by laser prepulses. Staging LWFA (Injector booster scheme) had been demonstrated. A new LWFA Platform at SPring-8/SACLA is under construction, which will enable them to test Staging LWFA in full scale.

To improve the acceleration quality, detailed acceleration structure should be measured and controlled. In the talk given by Hua [L-I8], it was reported that a high-energy (70 MeV) fs LWFA electron beam can be used to probe the field structure of plasma wake. The highly transient, microscopic wakefield is reconstructed from the density-modulated ultrashort probe bunch after it has traversed the wake. This technique enables visualization of wakefield and its evolution in low-density plasmas, and is a new diagnostic tool for the study of wakefield acceleration (Zhang et al. 2017).

Li and Leng [L-19] reported their progresses on LWFA at Shanghai Institute of Optics and Fine Mechanics (SIOM), CAS. Based on the front end of $10 \mathrm{PW}$ laser, they built a $200 \mathrm{TW} / 1-5 \mathrm{~Hz}$ Ti: sapphire laser system with good performance for driving LWFA. In particular, they had demonstrated the generation of high-brightness electron beams from a 2-stage LWFA, the maximum 6-D brightness $\sim 6.5 \times 10^{15} \mathrm{~A} / \mathrm{m}^{2} / 0.1 \%$ with charge $10-80 \mathrm{pC}$ in the energy range of $200 \sim 600 \mathrm{MeV}$ (Wang et al. 2016), which is comparable with the state-of-the-art LINAC drivers. Based on the high-quality electron beams, Compton scattering gamma rays were obtained with a peak brilliance of $\sim 3 \times 10^{22}$ photons $\mathrm{s}^{-1} \mathrm{~mm}^{-2}$ $\operatorname{mrad}^{-2} 0.1 \% \mathrm{BW}$ at $1 \mathrm{MeV}$ (Yu et al. 2016a, b). Furthermore, LWFA-based soft $\mathrm{X}$-ray FEL at $30 \mathrm{~nm}$ is in progress with undulator emission measured and a $6 \mathrm{~m}$ long TGUundulator is installed. Some of their other activities related to the development of multi-PW high-power laser systems were also introduced as described in Sect. 5 of this report.

Chen [L-I18] reported their efforts on electron injection control in LWFA to obtain high-quality beam acceleration via ionization injection, first proposed by their group in 2006 (Chen et al. 2006). Two optimized ionization injection schemes had been proposed and demonstrated via numerical simulation. By use of certain initially unmatched laser pulses, the electron injection can be constrained to the very front region of the mixed gas target, typically in a length of a few hundreds of micro meters determined by laser-driven bubble deformation. As a result, the final electron energy spread is largely reduced. It is called self-truncated ionization injection (Zeng et al. 2014), which was demonstrated experimentally in Shanghai Jiao Tong University (Mirzaie et al. 2015; Hafz et al. 2016). In the second scheme, using two laser pulses with fundamental frequency and high harmonics co-propagating in a gas target, ionization injection can be further controlled by proper choice of the two laser field amplitudes. Due to the phase velocity difference of the two color pulses, the injection length can be controlled within a short distance of few tens of micrometers. Electron beam with ultralow energy spread less than one percent was demonstrated numerically (Zeng et al. 2015).

Zhang [L-I20] presented an X-ray laser-driven wakefield accelerator in a nanotube to further increase the acceleration gradient as compared to normal LWFA driven by lasers in optical wavelength. It has been proposed before that electron acceleration gradients can be much enhanced to the $\mathrm{TeV} / \mathrm{cm}$ level when the wakefield is driven 
in metallic crystal (Chen et al. 1986; Tajima and Cavenago 1987). Here the wakefield is induced by a coherent, ultrashort X-ray pulse guided by a nanoscale channel inside a solid material. Two-dimensional particle-in-cell simulations show that an acceleration gradient of $\mathrm{TeV} / \mathrm{cm}$ is attainable, which is about three orders of magnitude higher than that of the normal plasma-based wakefield accelerations in lowdensity plasma. In addition to particle acceleration, the scheme can also produce high energy photons at tens of MeV (Zhang et al. 2016b).

Associated with LWFA in plasma, Sengupta [L-I7] presented their theoretical work on the breaking of relativistically intense longitudinal waves in plasma. They found that the wavebreaking occurs via a process called phase mixing. Phase mixing results in crossing of neighboring electron orbits due to the temporal dependence of phase difference between oscillating electrons. This temporal dependence of the phase difference between neighboring oscillating electrons can be caused by the background density inhomogeneity and/or relativistic mass variation effects. In particular, they found that the longitudinal Akhiezer-Polovin wave breaks via phase mixing at the amplitude well below its wavebreaking limit when it is longitudinally perturbed. Therefore, they believe that experiments depending on Akhiezer-Polovin wavebreaking limit for their interpretation may require revisiting. They further found that longitudinal waves in warm plasma also break via the process of phase mixing. A series of work had been published by their group on this topic (Verma et al. 2012; Sengupta et al. 2011; Mukherjee and Sengupta 2016a, b).

\subsection{Laser plasma-based radiation and plasma photonics}

Based upon LWFA-produced electron beams, X- and gamma rays can be produced in plasma. Significant efforts have been made to improve their controllability, including the peak brightness, spectrum tunability, etc. In the same talk by Chen [L-I18] mentioned above, an all-optical synchrotron-like radiation source was discussed based on laser plasma acceleration either in a straight or in a curved plasma channel. With the laser pulse off-axially injected in a straight channel, the centroid oscillation of the laser pulse causes a wiggler motion of the whole accelerating structure including the trapped electrons, leading to strong synchrotron-like radiations with tunable spectra and with controllable polarization of the produced X-rays (Chen et al. 2016; Luo et al. 2016). It was further shown that a ring-shaped synchrotron was possible in a curved plasma channel. Due to the intense acceleration and bending fields inside plasmas, the central part of the sources could be made within palm size, which is attractive for applications. Recent studies suggest that a curved plasma channel can be applied to staged LWFA for high-energy electron accelerators (Luo et al. 2018).

Besides wakefield-based X-ray radiation sources, Qiao [L-I30] presented their studies on the brilliant gamma ray emission from near-critical plasma with ultraintense laser pulses. A novel resonant acceleration scheme was found for generating dense relativistic electron bunches and emitting brilliant $\gamma$-ray pulses (Liu et al. 2013, 2015), where the laser frequency matches that of electron betatron oscillation under quasi-static electromagnetic fields in plasma. 3D PIC simulations show that 
brilliant $\gamma$-ray radiation with energy of $3 \mathrm{~J}$ and brightness of $10^{24}$ photons $/ \mathrm{s} / \mathrm{mm}^{2} /$ $\operatorname{mrad}^{2} / 0.1 \% \mathrm{BW}$ (at $3 \mathrm{MeV}$ ) can be produced using circularly polarized lasers at intensity $10^{22} \mathrm{~W} / \mathrm{cm}^{2}$, where the induced quasi-static longitudinal magnetic fields play a significant role (Zheng et al. 2005). It was found that the total number of radiated photons scales as $a^{2} /(S)^{1 / 2}$ and the conversion efficiency scales as $a^{3} / S$, where $S=\left(n_{\mathrm{e}} / n_{\mathrm{c}}\right) a$ and the variable $a$ is the laser-normalized amplitude (Chang et al. 2015, 2017). Further studies show that if the laser intensity is increased to $10^{23} \mathrm{~W} / \mathrm{cm}^{2}$, the quantum electrodynamic (QED) effects are favorable for electron trapping and resonance acceleration, resulting in production of brilliant $\gamma$-ray pulses with brightness of $10^{25}$ photons $/ \mathrm{s} / \mathrm{mm}^{2} / \mathrm{mrad}^{2} / 0.1 \%$ BW at $15 \mathrm{MeV}$ (Huang et al. 2017a, b).

Huang [L-I33] reported theoretical investigation on gamma ray production driven by ultra-intense lasers, where QED effect becomes significant (Huang 2015). The combination of ultra-intense lasers, the conventional accelerators and nuclear physics will provide a large scientific research platform for laser-nuclear physics. He also proposed the laser Compton scattering gamma ray source based on the BEPCII facility in Beijing, which could be applied in X-ray/ $\gamma$-ray calibration, photon nuclear physics, nuclear astrophysics, $\gamma-\gamma$ collider, and basic QED/QCD physics.

In the last decade, there has been significant interest in the topic of plasma photonics, in which various plasma density structures were adopted to control the laser propagation for different applications from laser-driven particle acceleration to laser fusion. A few novel concepts have been proposed, such as plasma optical amplifiers (Shvets et al. 1998; Malkin et al. 1999; Ping et al. 2000; Trines et al. 2011; Vieux et al. 2011; Weber et al. 2013), plasma mirror (Thaury et al. 2008), plasma channel or lens (Esarey et al. 1996; Milchberg et al. 1996; Ting et al. 1997; Wang et al. 2011; Chen et al. 2016), plasma grating (Sheng et al. 2003; Wu et al. 2005a, b; Yu et al. 2009), plasma optical modulator (Yu et al. 2016b), plasma polarizer (Weng et al. 2017; Wang et al. 2015c), etc.

Related to plasma photonics, Pai [L-I4] reported a method for fabricating transient plasma structures with high-intensity laser pulses. Such plasma structures are useful for better control of laser-plasma interaction (Pai et al. 2005). Programmable fabrication of longitudinal spatial structures in a gas jet was achieved using laser machining with a liquid-crystal spatial light modulator as the pattern mask (Lin et al. 2006). These structures were used as programmable photonic devices in the development of laser-driven particle accelerators and plasma nonlinear optics driven by multi-terawatt lasers. Periodic plasma structures were used to achieve quasi-phase matching in relativistic harmonic generation (Kuo et al. 2007). By scanning the interaction length with the same method, tomographic measurements were carried out to resolve the injection/acceleration process in laser wakefield accelerators and the amplification processes in plasma nonlinear optics (Pai et al. 2006; Hsieh et al. 2006; Chang et al. 2007; Hsieh et al. 2008). By adding a transverse heater pulse into the axicon-ignitor-heater scheme for producing a plasma waveguide, a variable three-dimensionally structured plasma waveguide was fabricated (Hung et al. 2012). Applications of laser-fabricated plasma structures in plasma nonlinear optics, particle acceleration and ultra-intense mid-infrared pulse generation were also discussed (Pai et al. 2010). 
Plasma mirrors are found to be an indispensable optical element for high-power laser systems, in particular, when the laser peak power is at the PW scale or above. Lee [L-O3] reported the double plasma mirror system developed for their $4 \mathrm{PW}$ laser system to increase the laser pulse contrast. Reflectivity of the double plasma mirror was measured as $\sim 70 \%$ which shows better performance compared to the reflectivity measured with $1 \mathrm{PW}$ laser system of $\sim 40 \%$. Moreover, to avoid damage of some important equipment from the back-reflected light, they tested some configurations of two plasma mirrors.

\subsection{Laser-driven ion acceleration and applications}

Laser-driven ion acceleration has attracted a great deal of interest in the last two decades due to its great potential of broad applications ranging from fundamental research to medical and industrial applications. Well-known mechanisms of ion acceleration include target normal sheath acceleration (TNSA), collisionless shock acceleration, radiation pressure acceleration (RPA), Coulomb explosion, and the combination of these mechanisms. Significant progress has been made in this topic (Daido et al. 2012; Macchi et al. 2013). However, to realize real applications, more efforts are still required to improve the ion beam quality, e.g., enhancing the maximum energy, reducing the beam energy spread, increasing the stability and controllability (Schreiber et al. 2014; Wagner et al. 2014).

In his talk, Yan [L-I13] reported their experimental efforts in this direction. By integrating a laser ion acceleration device and beam transportation system, a plasma ion accelerator was constructed at Peking University recently. Proton beams with energy of 3-9 MeV, energy spread less than $1 \%$ and charge of 1-20 pC were obtained from this facility, where the proton energy stability can be smaller than $3 \%$ (Shou et al. 2017). This makes the laser plasma-based ion therapy more close to reality. Moreover, in their recent collaboration experiment with CoReLS in Gwangju in Korea using the PW laser in CoReLS, they had achieved 0.6 GeV carbon acceleration in cascaded acceleration by combining the RPA and TNSA mechanisms.

Murakami [L-I26] reported theoretical and numerical investigation on quasimonoenergetic proton generation for compact neutron sources. By irradiating ultraintense ultrashort laser pulses on nanosized cluster targets, protons were accelerated to a few MeV due to Coulomb explosion (Murakami and Mima 2009). In particular, the cluster targets were made of two or three atomic components to produce quasimonoenergetic protons. The 50-100 nm sized spherical targets were optimized in structure to provide a quasi-monoenergetic proton source, where the conversion efficiency from absorbed energy to proton kinetic energy amounts was as high as $30 \%$. Neutrons are generated via the reactions between lithium and the protons. Optimizing the laser and target parameters, they maximized the coupling efficiency of neutron yields. A most salient feature of the optimized target is the hollow structure. As long as the quasi-monoenergetic proton beam overlapped with the peak of $\mathrm{p}-\mathrm{Li}$ reaction rate, one can maximize the neutron production rate. The resultant neutrons are expected to have relatively low temperatures that are lower than a few $100 \mathrm{keV}$ because of the endothermic reactions. 
Krishnmaurthy [L-I27] reported on the acceleration of neutral atoms in laserproduced plasmas. It was shown that laser plasma ion accelerators can be converted to compact-efficient MeV neutral atom accelerators (Rajeev et al. 2013, 2015; Malay et al. 2017). In particular, with nanocluster exposed to intense laser pulses, it is possible to convert more than $90 \%$ of the accelerated ions to neutral atoms. Furthermore, Krishnmaurthy showed that even traditional ion generation mechanism like TNSA could be optimized to convert most of the fast ions to neutrals. The advantage of high brightness-low emittance of ion generation from ultrashort high-intensity laser plasma formed with solid targets are known and a neutral atom source with the same emittance characteristics is inconceivable with conventional techniques of fast atom generation. Thus, this finding is an important addition to the field of ion acceleration from intense laser-produced plasmas.

Yogo [L-I17] reported on ion acceleration mechanism driven by multi-picosecond PW laser pulses. They demonstrated that high-contrast multi-picosecond pulses were advantageous for proton acceleration. Laser-driven ion acceleration with the laser intensity ranging from $10^{18}$ to $10^{22} \mathrm{~W} / \mathrm{cm}^{2}$ is predominantly governed by the absorption mechanism of laser energy into hot electrons. In their experiment, it was found that the plasma electrons were heated beyond the typical scaling law (Yogo et al. 2017), which is often considered to be at the ponderomotive potential (Kemp and Divol 2012). The experiment was performed using LFEX laser of Institute of Laser Engineering (ILE), Osaka University, which provides four beams of $1.5 \mathrm{ps}$ (FWHM) duration. By setting intervals of 1.5 ps between the four pulses, the overall pulse duration becomes 3 ps when two pulses are used and it becomes 6 ps when four pulses are used. The maximum laser energy in total was $1 \mathrm{~kJ}$ (250 J for each laser beam) and the intensity was $2.3 \times 10^{18} \mathrm{~W} / \mathrm{cm}^{2}$ on the target. The focal spot diameter was set to be $60 \mu \mathrm{m}$ (FWHM), which made a beneficial effect on the electron recirculation around the target. When they expanded the pulse duration of the laser from 1.5 to 3 ps at the fixed laser intensity, the electron temperature was drastically enhanced up to $1.1 \mathrm{MeV}$, exceeding the ponderomotive potential around $0.2 \mathrm{MeV}$ for the laser intensity of $2.3 \times 10^{18} \mathrm{~W} / \mathrm{cm}^{2}$. By extending the pulse duration from 1.5 to 6 ps with fixed laser intensity of $10^{18} \mathrm{~W} / \mathrm{cm}^{2}$, the maximum proton energy was improved more than twice (from 13 to $33 \mathrm{MeV}$ ). The proton energies observed were discussed using a plasma expansion model with enhanced electron temperature beyond the ponderomotive potential.

Related to the work reported by Yogo, Morace [L-I5] reported on further experimental investigation on electron and proton acceleration by use of the LFEX-GXII laser facility. They described a method to enhance the laser energy absorption into fast electrons without significant improvement of the laser beam specifications through energy and intensity upgrades. By combining four beamlets with a small angle, hence having the interference patterns at the overlapping point, the laser absorption and the conversion efficiency from laser to hot electrons were much better than those with only one beam under the same laser energy and intensity. The four LFEX laser beamlets were focused on a $5 \mu \mathrm{m}$ Al foil followed by a single beamlet focused on the same type of target in different shots. A significantly higher hot electron temperature and proton peak energy, as well as laser-to-electron/proton 
energy conversion efficiency were measured in case of the four interfering beamlets. The strong azimuthal magnetic fields induced during the laser interaction dephase and de-couple the oscillating electrons from the laser field, thus improving the absorption into fast electrons and protons.

Lee [L-I15] from Korea Atomic Energy Research Institute reported a new type of layered target for the generation of an energetic proton beam with narrow energy spread. In this target, the ion layer was embedded inside the foil target and ion acceleration if realized by utilizing a bulk electrostatic field in the plasma (Kim et al. 2016). Under optimized target parameters, protons can be accelerated to around $300 \mathrm{MeV}$ with quasi-monoenergetic spectrum under the peak laser intensity of $10^{21} \mathrm{~W} / \mathrm{cm}^{2}$ based upon their numerical simulation. From their experiments carried out under the laser intensity of $10^{19} \mathrm{~W} / \mathrm{cm}^{2}$ with the sandwich target of $\mathrm{Cu}$ $6 \mu \mathrm{m}+\mathrm{PC} 2 \mu \mathrm{m}+\mathrm{Cu} 0.5 \mu \mathrm{m}$, they found some experimental evidence of the bulk acceleration. Future experiments are planned with lasers at higher peak power.

\section{High-energy density physics and laboratory astrophysics driven by lasers}

Usually high-energy density states of matter are obtained either using strong shocks from kJ ns-laser pulses or through isochoric heating of matter using short-pulse intense lasers. These two approaches are complementary: laser-driven shocks allow getting high densities (above solid state density) but relatively low temperatures (usually a few eV or a few $10 \mathrm{eV}$ at most); Instead, short-pulse isochoric heating is limited to solid density but can achieve much higher temperatures. Such states of matter can be found in different astrophysical environments and in different schemes of laser fusion. These enable one to explore the extreme rich physics otherwise not easily accessible Drake 2006; Remington et al. 2006; Ichimaru 2017).

The plenary talk by Li [P 30] presented an overview of laboratory astrophysics studies in China by use of high-power lasers. The first topic of their studies was on the magnetic reconnection (MR), which is believed to exist widely in space and astrophysical environment in different parameters. By use of quasi-static magnetic fields produced by high-power laser pulses, they reconstructed the topology of magnetic reconnection using the SG-II laser facility in Shanghai and the Gekko XII laser facility in Osaka. By use of laser-irradiated coil targets, they were able to produce strong magnetic field as high as hundreds of Tesla (Zhu et al. 2015), which allowed them to investigate MR in various magnetic field configurations. Ejection of energetic electrons with a spectrum similar to that from solar flares was observed (Zhong et al. 2016). The second topic was about collisionless shock waves. It is supposed that many astronomical and astrophysical shock waves are collisionless. In their investigation, the collisionless shockwaves were generated by two counterstreaming laser-produced plasmas (Liu et al. 2011). Numerical simulations indicate that the shockwaves are excited by electrostatic instability. The formation of plasma filaments was also observed, which is believed to be caused by the Weibel instability. Third, they investigated plasma jet formation and propagation. In particular, they observed a large angle deflection of the jets when two high-density plasmas jets 
propagate perpendicular to each other (Yuan et al. 2015; Zhao et al. 2016), which could be relevant to some astrophysical observation, e.g., the HH110/270 system.

In his invited talk, Koenig [L-I1] presented their recent radiative hydrodynamic experiment on laboratory astrophysics. This is a joint investigation with groups from Japan, UK, and USA. For more than a decade, they have performed laboratory experiments in connection with astrophysical phenomena, to improve the understanding of radiation hydrodynamics (Vinci et al. 2005) and to validate numerical schemes and assumptions in simulations. Their recent experiments focused on highly radiative shocks in interaction with solid obstacles (Koenig et al. 2017) (impact for molecular clouds physics) and Rayleigh-Taylor instabilities (RTI) that play a major role in astrophysical fluid dynamics. First, the highly radiative shocks in their experiments were generated in a low-density gas-filled cell obtained on the GEKKO XII laser facility. The shock waves were generated using an ablator-pusher target $(\mathrm{CH} / \mathrm{Au} / \mathrm{Ti})$, designed to limit as much as possible the preheating produced by the hot corona. The propagation media was Xe or He gas, with the aim to compare radiative effect in each medium. High-velocity shock waves were generated $(100-140 \mathrm{~km} / \mathrm{s})$ with a clear influence of a radiative precursor on a spherical obstacle mimicking interaction with molecular clouds. Regarding the RTI instabilities, they might be responsible for the absence of spherical symmetry in the shape of the supernova remnant, and might affect the dissipation of the energy. Currently, models are often not accurate enough, as even the late-time behavior of a single-mode RTI is not well-known. In this context, they performed an experiment on LULI2000 to deepen our knowledge on highly nonlinear RTI. The two media for the development of the instabilities were, respectively, brominated plastic and low-density foam. The interface was initially modulated $(120 \mu \mathrm{m}$ wavelength with $20 \mu \mathrm{m}$ in amplitude). X-Ray radiograph using Pico2000 (80 J, 1 ps) was performed. In both cases, 2D radiative hydrodynamic simulations were performed with the FLASH code developed at Univ. Chicago.

Cho [L-I2] from GIST in Korea reported the study of warm dense plasmas with ultrafast X-rays. Series of time-resolved X-ray spectroscopy experiments were performed to investigate thermal properties and bonding of several elements in warm dense matter (WDM) conditions, where the thermal energy is comparable to the Fermi energy and the ions are strongly coupled. For warm dense $\mathrm{Cu}$, the electron-ion relaxation was determined experimentally, and compared with various theoretical models. The electron-phonon couplings were temperature dependent and enhanced by a factor of 4-6 compared to previously known value (Cho et al. 2016). For $\mathrm{SiO}_{2}$, dynamics of $\mathrm{Si}-\mathrm{O}$ bond braking and a creation of electronic states within the band gap were observed. These provide a description of how $\mathrm{SiO}_{2}$ transforms from an insulator with well-defined local structure to a high-temperature liquid with reduced $\mathrm{Si}-\mathrm{O}$ bonding (Engelhorn et al. 2015).

Jakubowska [I-I32] reported the generation of high pressure in aluminum by femtosecond laser irradiation. The talk reported about results obtained in a recent experiment at the LOA using a Ti:Sa laser for the creation of strong shocks using short-pulse lasers. The produced shock pressure can initially be in excess of 100 Mbar even if it is not maintained in time due to the short duration of the laser pulse. Indeed, the generated shock immediately becomes a blast wave, i.e., a pressure 
pulse that travels in the material and gradually decreases its pressure. Blast waves are important in physics being present in a variety of phenomena: nuclear explosions, astrophysics (i.e., supernovae explosions), and hydrodynamics. For this reason, blast waves generated by short-pulse relatively high-intensity lasers had already been studied in gases. Works had focused on generating high Mach number blast waves (Edens et al. 2004), studying deflagration and detonation (Yoh et al. 2008) and realizing experiments in the framework of laboratory astrophysics (Moore et al. 2005). Blast waves were also observed in solids (Budil et al. 2000) using a very high-energy petawatt laser at Livermore, where the involved mechanism was not discussed in detail. In the experiment by Jakubowska and collaborators, a laser system delivering $3 \mathrm{~J}$ in $24 \mathrm{fs}$ pulses with a $10^{8}$ contrast was focused at $I \sim 10^{21} \mathrm{~W} / \mathrm{cm}^{2}$ on Al flat targets of different thicknesses 3, 6, 10, 15, and $50 \mu \mathrm{m}$. A streak camera was looking at the emission from target rear side. The analysis of the streak camera signal reveals a signal with three different phases: (1) a rapid emission following the passage of the hot electrons (due to thermal heating and to optical transition radiation), (2) a thermal decay due to plasma expansion and cooling, and (3) a final thermal emission peak. The transit time between two emission peaks corresponds to a supersonic velocity, supporting the evidence for shock wave generation. The work done to analyze the experimental results was divided into two steps. In the first step, a semi-analytical model was used to calculate the energy deposition of hot electrons in the target and the building of the temperature gradient, taking into account hot electron production by the relativistic intense lasers and hot electron transport. In the second step, this temperature gradient was used as input parameter for 2D hydrodynamics simulations performed with the code CHIC (Maire et al. 2007; Breil et al. 2007). Simulations show different interesting phenomena like the propagation of the blast wave, and the rapid expansion of the material due to the hot electron-induced strong preheating. The two effects, reduction of density (implying an acceleration of the shock wave) and reduction of blast wave pressure (implying a deceleration) compensate each other resulting in a blast wave moving at constant velocity in the expanding material.

Batani [L-O6] reported their work on the refraction index of shock-compressed water in the megabar pressure range. The study of matter in extreme states is interesting from many fields of physics going from laboratory astrophysics to inertial confinement fusion (Batani 2016). One particularly interesting material in the field planetological research is water, which is one of the main components of the mantles of giant planets like Uranus and Neptune (as well as of many recently discovered extra-solar planets). The observation of large and non-symmetric magnetic fields of Uranus and Neptune has attired interest toward the phase diagram of water at very high pressure, and the possible transitions towards conducting states (either metallic or superionic). In this context, they presented results on water with different phases and the variation of its refraction index as pressure and temperature increases (due to the action of laser-driven shock waves) (Batani et al. 2015). Results for the refraction index of water can be grouped into three different categories: lower pressure and temperature (approximately $P \sim 0.4 \mathrm{Mbar}$ and $T \sim 0.2 \mathrm{eV}$ ), higher pressure and temperature $(P \sim 1 \mathrm{Mbar}, T \sim 0.5 \mathrm{eV})$, and high pressures at lower temperature $(P \sim 1.2 \mathrm{Mbar}, T \sim 0.3 \mathrm{eV})$. The experimental results were compared to theoretical 
calculations performed at CEA via the quantum molecular dynamic calculations with the ABINIT-ATOMPAW code (Holzwarth et al. 2007). The optical conductivity was obtained by applying the Kubo-Greenwood formula (Mazevet et al. 2010). Theoretical calculations produce results in the same ranges of observed experimental results and are in qualitative agreements with them. Finally, they interpolated theoretical and experimental results using an extended Lorentz-Drude model.

Short-pulse-induced hydrodynamic can be roughly divided into two regimes (Pasley et al. 2017): (1) hydro driven directly by the laser [e.g., hole boring/radiation pressure (RP) driven]; or (2) hydro driven indirectly by pressure gradients induced by heating. It is relevant to inertial fusion energy (IFE) in the areas addressing fast ignitor hotspot, fast ignitor cone-tip physics and structured collimators. In addition, short pulses enable creating extreme conditions, which may be relevant to issues in IFE and elsewhere in HED physics: (a) ICF hotspot dynamics, (b) studies of hightemperature opacity and EOS. In these cases, the hydro may or may not be a desirable feature of the short-pulse interaction. Understanding the hydrodynamic behavior under the influence of a short pulse is however vital to designing such experiments. A range of different hydrodynamic effects may arise due to the illumination of a target with a short-pulse laser. Hydrodynamics in dense plasmas at sub-RP intensities is driven predominantly by pressure gradients. Modeling is challenging requiring a range of different codes to tackle the different stages of the interaction (prepulse, main pulse, later motion). In the meanwhile, few diagnostics are capable of resolving hydrodynamic behavior occurring on picosecond timescales. In addition, highenergy short-pulse laser plasma interactions generate intense competing "noise" signal, further complicating diagnosis. Pasley [L-I28] presented their study of the hydrodynamics induced on thin solid targets by the irradiation with ultra high-intensity short-pulse lasers. This work was carried out through collaboration with collaborators in TIFR in India and RAL in the UK. At TIFR, short-pulse-driven hydro has been measured directly in the lab with a pump-probe configuration, using $400 \mathrm{~nm}$ probe to record hydrodynamics driven by $800 \mathrm{~nm}$ pump beam (30 fs) at moderately high intensity $\left(5 \times 10^{16}\right.$ to $\left.1.5 \times 10^{17} \mathrm{~W} / \mathrm{cm}^{2}\right)$. This has allowed showing the generation of strong shocks and also how shock strength depends on the level of laser prepulse (therefore we can "control" shock strength by changing the prepulse). Markedly higher velocities achieved in the case of high-contrast interaction (Adak et al. 2017). The generation of acoustic waves was observed with Doppler spectroscopy measurements with a temporal resolution of around 100 femtoseconds. Measurements with very high temporal resolution reveal unexpected oscillatory behavior in the reflectivity. Combined reflectivity and Doppler measurements could be explained by an approximately sinusoidal density disturbance propagating down the density gradient through the probed density contour (Adak et al. 2015). The source of such a disturbance at the appropriate frequency (hundreds of $\mathrm{GHz}$ ) was found by looking at simulation results. Simulations revealed that the prompt heating of the preformed plasma (picosecond contrast is $\sim 10^{-4}$, nanosecond contrast is $\sim 10^{-8}$ ) results in a velocity flow gradient. This drives the acoustic modulations.

In experiments carried at RAL using the VULCAN PW laser at $I \sim 2.5 \times 10^{20} \mathrm{~W} /$ $\mathrm{cm}^{2}$ (duration $700 \mathrm{fs}$, energy $300 \mathrm{~J}$ ) and planar target (three layers: $\mathrm{X} \mu \mathrm{m} \mathrm{CH}-0.2 \mu \mathrm{m}$ $\mathrm{Al}-4 \mu \mathrm{m} \mathrm{CH}$ where the thickness of the front layer was varied between 4 and 
$64 \mu \mathrm{m}$ ), the emission from the rear side of the target was measured (not directly illuminated by the laser) in the XUV range using XUV multilayer mirrors (Lancaster et al. 2017). Emission shows a characteristic "holey" shape, particularly strong in the case of targets with $4 \mu \mathrm{m} \mathrm{CH}$ at front. Calculations show that the formation of a ring-like emission pattern is a stable feature of these experiments. Different codes have been used for the rad-hydro modeling (Hyades, h2d and FLASH). They show that the ring-like emission is most pronounced (and similar to the experiment) when a compression wave is formed: pressures in these waves can run to hundreds of Mbar. Also, the radius of the ring-like emission structure formed is dependent on injection properties of the electron beam.

$\mathrm{Hu}$ [L-I29] reported their work on laser's interaction with magnetized plasma. A magnetized laser plasma facility was built at University of Science and Technology of China (USTC), which is composed of a nanosecond heating laser beam $(6 \mathrm{~J} / 527 \mathrm{~nm} / 7 \mathrm{~ns})$, a femtosecond detecting laser beam $(2 \mathrm{~mJ} / 800 \mathrm{~nm} / 50 \mathrm{fs})$, and a 7-30 T pulsed magnetic field generator ( $\mathrm{Hu}$ et al. 2015). A series of laser plasma evolution experiments were performed (Tang et al. 2018). First, when a high-speed plasma stream produced by laser ablated solid target expands in an external transverse magnetic field, an asymmetric hollow plasma bubble is formed. Hall MHD simulation shows that the asymmetry of the bubble is induced by the Hall effect. Second, the enhanced laser ablation by the external $7 \mathrm{~T}$ magnetic field was observed in plasma optical images. The target surface, which contacts the plasma bubble, is heated by the energy transport along the surface of the plasma bubble. Thus, the debris and the target surface surrounding the laser focus spot are ablated and increases the plasma generation. Finally, when an external magnetic field was added along the laser channel in underdense plasma, the electron thermal transport was found to be suppressed by the magnetic field, which enhances the plasma temperature. This may be applied to restrain the stimulated scattering process in laser fusion. They found that 20-30 T external magnetic field was needed to mitigate the stimulated scattering process on China's SG-III prototype laser facility.

$\mathrm{X}$-ray diagnostics of high-energy density states of matter are critical in this area. Zhao [L-O2] reported an experimental study of K-shell absorption spectra in dense plasma. Highly compressed warm dense matter and hot dense plasma are relevant to the states of matter in planetary interiors and inertial confinement fusion. In their work, X-ray radiation-driven shocks driven by the SG-II laser facility were adopted to generate the dense plasma under high pressure (Zhang et al. 2012; Zhao et al. 2013, 2017). In their experiment on warm dense matter, colliding shocks driven by laser-converted radiation were used to compress aluminum/silicon to over twice solid density and low temperature below $1.0 \mathrm{eV}$. X-ray absorption spectra were obtained, which provided direct information about the density, temperature, electronic, and ionic structures of dense matter. The measured K-shell edge was compared to quantum molecular dynamics calculations. In their hot dense plasma experiment, the aluminum sample was heated by M-band emission and compressed by radiation-driven shocks. The time-resolved $1 \mathrm{~s}-2 \mathrm{p}$ absorption features from F-like to $\mathrm{C}$-like ions were observed in series with the radiation ablating.

Pikuz [L-O1] reported new diagnostics development for pump-probe experiments, which combine pulsed power lasers as pump and XFEL beams as probe 
to investigate the transient behavior of solid materials such as lattice dynamics. This requires proper monitoring of shock wave strength, stability of shock wave generation, initial structure of used targets and homogeneity of XFEL probe beam. A few methods have been proposed and tested to control these parameters (Pikuz et al. 2016; Ruiz-Lopez et al. 2017).

Huang [L-O4] reported simulation studies on electron energy spectrum produced in magnetic reconnection in laser-produced plasma. Based upon their 2D PIC simulation, it was found that magnetic reconnection in laser-produced plasma can be divided into two stages, squeezing stage and reconnection stage (Lu et al. 2016; Huang et al. 2017a, b). In the first stage, due to the expanding and squeezing of the two plasma bubbles, the toroidal magnetic field is enhanced, especially in the colliding region. The magnetic field enhancement leads to a large Alfven speed, which is responsible for the rapid reconnection in the second stage. Two kinds of electron acceleration mechanisms are involved in the two stages, the betatron mechanism and the Fermi-like acceleration. After these acceleration processes, the electron energy spectrum shows a Maxwellian distribution at low energy and a power-law distribution at high energy.

Under very different laser parameters from those studies mentioned above in this section, Ji [L-I31] reported their investigation on near-QED regime of laser interaction with overdense plasma. Laser plasma interaction in the near-QED regime $\left(10^{22}-10^{25} \mathrm{~W} / \mathrm{cm}^{2}\right)$ exhibits exotic features other than relativity. While this regime is not yet accessible in laboratories at the moment, PIC simulation provides the most powerful tool to explore the new physics therein. They did full 3D PIC simulations to systematically investigate the interaction process at such extreme intensities and found that radiation of electrons in the laser field could fundamentally change the particle dynamics (Ji et al. 2014a, b, c). First, electrons transfer a significant fraction of their energy to radiation, producing $\mathrm{MeV}$ gamma photons at a very high efficiency. Thus, the laser energy absorption channel is notably tuned in a way that electrons get less portion of energy from the laser while photons obtain more, as the laser intensity rises. Second, electrons experience strong feedbacks due to radiation reaction (RR) force. They show that the RR force could be strong enough to compensate for the expelling laser ponderomotive force, so that electrons can be trapped inside the laser pulse instead of being scattered off. This anomalous trapping mechanism leads to a dense plasma bunch confined within the most intense region of the laser beam, which may be measured in experiments in the future. Furthermore, these confined electrons fiercely emit gamma photons at efficiencies up to $35 \%$. Therefore, ultra-bright gamma ray emission becomes the most prominent feature in such laser plasma interaction. The threshold for radiation reaction trapping of electrons is shown to be proportional to the cubic root of the ratio between the laser wavelength and the classical electron radius. In the presence of plasma, this threshold can be significantly lowered down to about $10^{23} \mathrm{~W} / \mathrm{cm}^{2}$. In a proper interaction geometry, they found that it might be possible to observe RR effect at $10^{22} \mathrm{~W} / \mathrm{cm}^{2}$ intensity level by doing laser electron collision using one single laser beam irradiating a micro-channel structure. 


\section{Development of high-power laser systems for relativistic laser plasmas}

High-power laser systems are critical for all applications from laser fusion, laserdriven particle acceleration, to high-energy density physics and laboratory astrophysics (Danson et al. 2015). In Asian countries, there are significant efforts in the development of various laser systems, including $100 \mathrm{~kJ}$ scale systems for laser fusion, multi-100 TW and even multi-PW laser systems for laser-driven particle acceleration and high-energy density physics.

In the talk by Li and Leng [L-I9] from SIOM in Shanghai, they reported new progress of implementing a 10 PW laser facility (SULF) in Shanghai, including the generation of a 5.4 PW 24 fs laser pulses from a Ti:Sapphire crystal-based CPA (chirped pulse amplification) laser system and the progress on LWFA towards high-quality electron beams. Earlier in 2015, the generation of $192.3 \mathrm{~J}$ laser pulse from a $150 \mathrm{~mm}$ in diameter Ti:Sapphire crystal was demonstrated (Chu et al. 2015). In 2016, a novel temporal dual-pulse scheme was developed to suppress the parasitic lasing (PL) and transverse amplified spontaneous emission (TASE). The maximum output energy of $202.8 \mathrm{~J}$ was obtained from the $150 \mathrm{~mm}$ diameter Ti:S booster amplifier with a pump energy of $320.0 \mathrm{~J}$, corresponding to a conversion efficiency of $49.3 \%$. The compressed pulse duration of 24.0 fs was measured with a throughput efficiency of $64 \%$, leading to a peak power of $5.4 \mathrm{PW}$ (Gan et al. 2017). The contrast is $10^{-10}$ about $50 \mathrm{ps}$ before the main pulse based on the single shot contrast measure method (Li et al. 2017b). In the end of 2016, this temporal dual-pulse pump technique was used in the $235 \mathrm{~mm}$ Ti:Sapphire to generate $339 \mathrm{~J}$ laser pulses, which is the largest Ti:Sapphire crystal and maximum output energy from Ti:Sapphire amplifier. It was estimated that it could support the $10 \mathrm{PW}$ peak power output. Besides the $10 \mathrm{PW}$ laser project based on Ti:Sapphire crystal, they also had demonstrated a 1 PW OPCPA laser amplifier based on a $100 \mathrm{~mm}$ size Lithium triborate (LiB3O5 or LBO) crystal (Yu et al. 2015). After optimization, an amplified energy of $45.3 \mathrm{~J}$ was achieved with a conversion efficiency of $26.3 \%$ in OPCPA stage. The peak power of the compressed pulse was $1.02 \mathrm{PW}$ with a compressed duration of $32 \mathrm{fs}$ (Yu et al. 2016a, b). Based on the front end of 10 PW laser, they built a 200 TW/1-5 Hz Ti:Sapphire laser with good performance for driving LWFA (Xu et al. 2016, Wang et al. 2016) as mentioned in Sect. 3 in this report.

Kiriyama [L-I10] reported on the development of their high-contrast J-KAREN-P laser facility at Kansai Photon Science Institute in Japan. The J-KAREN-P laser facility can provide PW peak power at $0.1 \mathrm{~Hz}$ (Kiriyama et al. 2015). It is based on the generation of short pulses of $30 \mathrm{fs}$ and energy of $30 \mathrm{~J}$ after compression. The contrast of the generated pulses is better than $10^{12}$ and the final focused intensity is higher than $10^{22} \mathrm{~W} / \mathrm{cm}^{2}$. Such laser performance can lead to significant progress in physical experiments. The key features of the J-KAREN-P laser system architecture are: (1) the high-contrast front end source based on a combination of some saturable absorbers and low gain OPCPA configuration, (2) the main amplification composed of four moderate gain Ti:Sapphire 
amplifiers, employing simple off-axis beam expanders with low aberration, (3) the adaptive control of the residual spectral phase based on a high dynamic range Dazzler and the active wavefront correction based on a deformable mirror, (4) the main compressor stage based on four high-quality large-sized gold gratings. Using a $120 \mathrm{~mm}$ diameter Ti:Sapphire crystal and the total pump energy at a $0.1 \mathrm{~Hz}$ repetition rate, the maximum output energy of $63 \mathrm{~J}$ was achieved with an incident energy of $92 \mathrm{~J}$. The amplified pulses are up-collimated to $\sim 280 \mathrm{~mm}$ diameter and finally compressed in the compressor consisting of four 1480 grooves/mm gold-coated gratings of $565 \times 360 \mathrm{~mm}^{2}$. The measured spectrum has a bandwidth of $\sim 50 \mathrm{~nm}$ (FWHM). The obtained recompressed pulse duration is less than 30 fs. The peak power is expected to be over PW at $0.1 \mathrm{~Hz}$ on target. The contrast at less than 200 ps before the main pulse is $3 \times 10^{-12}$ (detection limited). With an f/1.3 off-axis parabolic mirror, according to measurements of the focal spot and energy contained within it, a peak intensity of $10^{22} \mathrm{~W} / \mathrm{cm}^{2}$ on target is achieved at the $0.3 \mathrm{PW}$ power level. The J-KAREN-P laser system at the National Institutes for Quantum Beam Science and Technology (QST) is one of the leading facilities in the provision and application of ultra high-intensity lasers for the broad community. This laser has been used in a variety of pioneering and cutting-edge research, which has resulted in high-impact discoveries for high field science (Kiriyama et al. 2015; Kando et al. 2009; Fukuda et al. 2013; Pirozhkov et al. 2014; Nishiuchi et al. 2015).

At CoReLS in the Institute for Basic Science in Gwangju, Korea, two PW laser beams (1 PW, $20 \mathrm{fs}$ ) are in operation with the repetition rate of $0.1 \mathrm{~Hz}$ and a $150 \mathrm{TW}$, $25 \mathrm{fs}$ Ti:Sapphire laser system is available with the repetition rate of $5 \mathrm{~Hz}$ as reported by Nam and Kim [L-I14, P-7], as also mentioned above in Sect. 3.1. They are used for various experiments including LWFA, ion acceleration, etc. Recently, one of the two PW beamlines has been upgraded to a 4 PW 20 fs laser to explore relativistic laser plasma interactions and strong field QED effects (Sung et al. 2017).

Kawanaka [L-I11] reported on their effort to develop high-energy laser pulses with high repetition rate. Here high energy usually means the pulse energy is over $10 \mathrm{~J}$ and high repetition rate means at $10 \mathrm{~Hz}$ or higher. Such laser systems are supposed to be important for many applications. The key challenge is that a high-energy laser pulse often require $\mathrm{Nd}$ :glass nanosecond laser as a pump source, which is hard to operate in high repetition rate. A few options to solve this challenge were discussed in his talk, including their "TRAM" design using cryogenic Yb:YAG ceramics (Furuse et al.2009) and "multi-TRAM" (Divoky et al. 2015).

\section{Concluding remark}

The Asian community in laser plasma has been growing rapidly partially owing to the development of high-power laser technologies, which bring an extremely bright prospect of laser plasma-based applications. One of the applications is plasmabased particle accelerators and radiation sources, which show unique properties and advantages compared with those based upon conventional technologies. Other applications include high-energy density physics and laboratory astrophysics, which are 
attracting increasing interest. 10-100 PW scale femtosecond laser systems and highenergy mega joule nanosecond laser systems are also under the construction or to be constructed in some Asian countries, which enable one to investigate laser plasma physics and applications in the unexplored new regimes. It appears that the field of laser plasma studies becomes more and more interdisciplinary, covering nonlinear optics, atomic physics, accelerator physics, nuclear physics, astrophysics, condensed matter physics, and QED physics. Asian research groups can make more significant contributions to this field with the prospects of more investment and more advanced facilities available in the Asian-Pacific region. More close collaboration among different groups and different countries in the Asian-Pacific region in this field is highly desirable in this area.

Acknowledgements I would like to thank the all speakers who presented their work at the laser plasma sessions of this conference, in particular, M. Chen, D. Batani, L. Ji, H. T. Kim, M. Koenig, K. Lan, X. Y. Leng, M. Murakami, J. Pasley, and W. M. Wang, who provided highly valuable assistance in producing this summary report.

\section{Compliance with ethical standards}

Conflict of interest I state that there is no conflict of interest concerning the contents in the report.

Open Access This article is distributed under the terms of the Creative Commons Attribution 4.0 International License (http://creativecommons.org/licenses/by/4.0/), which permits unrestricted use, distribution, and reproduction in any medium, provided you give appropriate credit to the original author(s) and the source, provide a link to the Creative Commons license, and indicate if changes were made.

\section{References}

Abstracts of 1st Asia-Pacific conference on plasma physics, plenary (http://aappsdpp.org/DPPProgram latest/wholeitem/Plenarysessions.html) and topical session (http://aappsdpp.org/DPPProgramlates t/topical_session.html). Names with presentation number are given for reference

A. Adak et al., Phys. Rev. Lett. 114, 115001 (2015)

A. Adak et al., Phys. Plasmas 24, 072702 (2017)

C. Aniculaesei et al., Rev. Sci. Instrum. 89, 025110 (2018)

S. Atzeni, J. Meyer-ter-Vehn, The Physics of Inertial Fusion (Oxford Science, Oxford, 2004)

M. Bailly-Grandvaux et al., Nat. Commun. 9, 102 (2018)

D. Batani et al., Physica Scripta T161, 014016 (2014)

D. Batani, K. Jakubowska et al., Europhys. Lett. 112, 49901 (2015)

D. Batani, Europhys. Lett. 114, 65001 (2016)

J. Breil et al., J. Comp. Phys. 224, 785 (2007)

K. Budil et al., Astrophys. J. 127, 261 (2000)

E.M. Campbell et al., Matter Radiat. Extremes 2, 37 (2017)

H. Cao et al., Phys. Plasmas 24, 082701 (2017)

A. Casner et al., High Energy Density Phys. 17, 2-11 (2015)

C.-L. Chang et al., Phys. Rev. E 75, 036402 (2007)

H.X. Chang, B. Qiao et al., Phys. Rev. E 92, 053107 (2015)

H.X. Chang, B. Qiao et al., Sci. Rep. 7, 45031 (2017)

M. Chen et al., J. Appl. Phys. 99, 056109 (2006)

M. Chen et al., Light Sci. Appl. 5, 16015 (2016)

P. Chen, R.J. Noble, A.I.P. Conf, Proc. 156, 222 (1986)

Y. Chen et al., Matter Radiat. Extremes 2, 77 (2017) 
Y. Chen et al., Phys. Plasmas 25, 022702 (2018)

B.I. Cho et al., Sci. Rep. 6, 18843 (2016)

Y. Chu et al., Opt. Lett. 40, 5011 (2015)

H. Daido, M. Nishiuchi, A.S. Pirozhkov, Rep. Prog. Phys. 75, 056401 (2012)

C. Danson et al., High Power Laser Sci. Eng. 3, e3 (2015)

M. Divoky et al., Opt. Lett. 40, 855 (2015)

R.P. Drake, High-energy-density physics: fundamentals, inertial fusion, and experimental astrophysics (Springer, Heidelberg, 2006)

H. Duan et al., Phys. Plasmas 22, 092704 (2015)

A.D. Edens et al., Phys. Plasmas 11, 4968 (2004)

C.B. Edwards, C.N. Danson, High Power Laser Sci. Eng. 3, e4 (2015)

S. Eliezer et al., Phys. Plasmas 23, 050704 (2016)

K. Engelhorn et al., Phys. Rev. B 91, 214305 (2015)

E. Esarey et al., IEEE Trans. Plasma Sci. 24, 252 (1996)

E. Esarey et al., Rev. Mod. Phys. 81, 1229 (2009)

Y. Fukuda et al., Radiat. Measur. 50, 92-96 (2013)

H. Furuse et al., Opt. Lett. 34, 3439 (2009)

Z. Gan et al., Opt. Express 25, 5169 (2017)

A. Giulietti, Laser-driven particle acceleration towards radiobiology and medicine (Springer Switzerland, 2016), https://doi.org/10.1007/978-3-319-31563-8_7

Y. Gu et al., High Power Laser \& Part. Beams 27, 27110101 (2015)

N.A.M. Hafz et al., High Power Laser Sci. Eng. 4, e24 (2016)

L. Hao et al., Phys. Plasmas 21, 072705 (2014)

X.T. He et al., Phys. Plasmas 23, 082706 (2016)

N. A. Holzwarth, M. Torrent, F. Jollet, ATOMPAW: pwpaw.wfu.edu; ATOMPAW2 ABINIT: www.abini t.org (2007)

H. Hora et al., Energy Environ. Sci. 3, 479 (2010)

H. Hora et al., Laser Part. Beams 32, 607 (2015)

H. Hora et al., High Power Laser Sci. Eng. 4, e35 (2016)

C.-T. Hsieh et al., Phys. Rev. Lett. 96, 095001 (2006)

C.-T. Hsieh et al., J. Korean Phys. Soc. 53, 3719 (2008)

G.Y. Hu et al., Plasma Sci. Technol 17, 134 (2015)

K. Huang et al., Phys. Plasmas 24, 041406 (2017a)

T.W. Huang et al., Appl. Phys. Lett. 110, 021102 (2017b)

Y.S. Huang, Sci. Rep. 5, 15866 (2015)

T.-S. Hung et al., Phys. Plasma 19, 063109 (2012)

W.Y. Huo et al., Phys. Plasmas 21, 114503 (2014)

W.Y. Huo et al., Matter Radiat. Extremes 1, 2 (2016a)

W.Y. Huo et al., Phys. Rev. Lett. 117, 025002 (2016b)

O.A. Hurricane et al., Nature 506, 343 (2014)

S. Ichimaru, Rev. Mod. Plasma Phys. 1, 6 (2017)

L.L. Ji et al., Phys. Rev. Lett. 112, 145003 (2014a)

L.L. Ji et al., Phys. Plasmas 21, 023109 (2014b)

L.L. Ji et al., Eur. Phys. J. Special Topics 233, 1069 (2014c)

M. Kando et al., Phys. Rev. Lett. 103, 235003 (2009)

M. Karasik et al., Phys. Rev. Lett. 114, 085001 (2015)

P.K. Kaw, Rev. Mod. Plasma Phys. 1, 2 (2017)

A.J. Kemp, L. Divol, Phys. Rev. Lett. 109, 195005 (2012)

M. Key et al., Phys. Plasmas 15, 022701 (2008)

K.N. Kim, K. Lee et al., Phys. Plasmas 23, 033119 (2016)

H.T. Kim et al., Phys. Rev. Lett. 111, 165002 (2013)

H.T. Kim et al., Sci. Rep. 7, 10203 (2017)

H. Kiriyama et al., IEEE J. Quantum Electron. 21, 1601118 (2015)

R. Kodama et al., Nature 412, 798 (2001)

M. Koenig et al., Phys. Plasmas 24, 082707 (2017)

C.-C. Kuo et al., Phys. Rev. Lett. 98, 033901 (2007)

K. Lan et al., Phys. Plasmas 21, 010704 (2014a)

K. Lan et al., Phys. Plasmas 21, 052704 (2014b) 
K. Lan et al., Phys. Plasmas 21, 090704 (2014c)

K. Lan, J. Liu, Z. Li et al., Matter Radiat. Extremes 1, 8 (2016)

K. Lan, Peng Song, Phys. Plasmas 24, 052707 (2017)

K. Lancaster et al., Phys. Plasmas 24, 083115 (2017)

K.F.F. Law et al., App. Phys. Lett. 108, 091104 (2016)

W.P. Leemans et al., Phys. Rev. Lett. 113, 245002 (2014)

M. Li, J.X. Wang et al., Phys. Plasma 24, 013117 (2017a)

S. Li, K. Lan, J. Liu, Laser Part. Beams 33, 731 (2015)

S. Li et al., Opt. Express 25, 17488 (2017b)

Z. Li, D. Yang, S. Li et al., Phys. Plasmas 24, 072711 (2017c)

J.D. Lindl, Phys. Plasmas 2, 3933 (1995)

M.-W. Lin et al., Phys. Plasma 13, 110701 (2006)

B. Liu et al., Phys. Rev. Lett. 110, 045002 (2013)

B. Liu et al., Phys. Plasmas 22, 080704 (2015)

X. Liu, Y.T. Li, Y. Zhang et al., New J. Phys. 13, 093001 (2011)

S. Lu et al., New J. Phys. 18, 013051 (2016)

J. Luo, M. Chen et al., Sci. Rep. 6, 29101 (2016)

J. Luo, M. Chen et al., Phys. Rev. Lett. 120, 154801 (2018)

A. Macchi et al., Rev. Mod. Phys. 85, 751 (2013)

P.-H. Maire et al., SIAM JSC 29, 1781 (2007)

M. Malay et al., Sci. Rep. 7, 3871 (2017)

V.M. Malkin et al., Phys. Rev. Lett. 82, 4448 (1999)

S. Mazevet et al., High Energy Density Phys. 6, 84 (2010)

H.M. Milchberg et al., Phys. Plasmas 3, 2149 (1996)

J.-L. Miquel, J. Phys: Conf. Ser. 717, 012084 (2016)

M. Mirzaie et al., Sci. Rep. 5, 14659 (2015)

Y. Mizuta et al., Phys. Rev. ST. 15, 121301 (2012)

A.S. Moore et al., Phys. Plasmas 12, 052707 (2005)

A. Morace et al., Proc. SPIE 10328, 103280 U (2017)

A. Mukherjee, S. Sengupta, Phys. Plasmas 23, 092112 (2016a)

A. Mukherjee, S. Sengupta, Breaking of large amplitude electron plasma wave in a Maxwellian plasma. ArXiv preprint arXiv 1605, 05518 (2016b)

M. Murakami, K. Mima, Phys. Plasmas 16, 103108 (2009)

N. Nakanii et al., Phys. Rev. ST 18, 021303 (2015)

M. Nishiuchi et al., Phys. Plasmas 22, 033107 (2015)

M. Nishikino et al., Phys. Plasmas 9, 1381 (2002)

R. Nora et al., Phys. Plasmas 21, 056316 (2014)

S.P. Obenschain et al., Phys. Plasmas 9, 2234 (2002)

C.-H. Pai et al., Phys. Plasma 12, 070707 (2005)

C.-H. Pai et al., Opt. Lett. 31, 984 (2006)

C.-H. Pai et al., Phys. Rev. A 82, 063804 (2010)

A. Picciotto et al., Phys. Rev. X 4, 031030 (2014)

A. Pikuz et al., J. Appl. Phys. 120, 035901 (2016)

Y. Ping et al., Phys. Rev. E 62, R4532 (2000)

A.S. Pirozhkov et al., New J. Phys. 16, 093003 (2014)

J. Pasley et al., AIP Conf. Proc. 1824, 020004 (2017)

R. Rajeev et al., Nat. Phys. 9, 185 (2013)

R. Rajeev et al., New J. Phys. 17, 023033 (2015)

B.A. Remington et al., Rev. Mod. Phys. 78, 755-807 (2006)

M. Ruiz-Lopez et al., J. Synchr. Rad. 24, 196-204 (2017)

J.J. Santos et al., J. Plasma Fusion Res. 93, 170-176 (2017)

J.J. Santos et al., New J. Phys. 17, 083051 (2015)

R. Sauerbrey et al., Phys. Plasmas 3, 4712 (1996)

S. Sengupta et al., Plasma Phys. Control. Fusion 53, 074014 (2011)

Z.-M. Sheng et al., Appl. Phys. B 77, 673 (2003)

H. Shiraga et al., Plasma Phys. Controlled Fusion 53, 124029 (2011)

Y. Shou, Y. Geng, Q. Liao et al., J. Phys: Conf. Ser. 874, 012043 (2017)

J. Schreiber, F. Bell, Z. Najmudin, High Power Laser Sci. Eng. 2, e41 (2014) 
Li Shu et al., Acta Phys. Sin. 64, 145203 (2015)

Li Shu et al., Acta Phys. Sin. 67, 025202 (2018)

G. Shvets et al., Phys. Rev. Lett. 81, 4879 (1998)

J.H. Sung et al., Opt. Lett. 42, 2058 (2017)

T. Tajima, J.M. Dawson, Phys. Rev. Lett. 43, 267 (1979)

T. Tajima, M. Cavenago, Phys. Rev. Lett. 59, 1440 (1987)

H.B. Tang et al., Plasma Phys. Control. Fusion 60, 055005 (2018)

C. Thaury et al., Nat. Phys. 4, 631-634 (2008)

W. Theobald et al., Phys. Plasmas 18, 056305 (2011)

V.T. Tikhonchuk et al., Phys. Rev. E 96, 023202 (2017)

A. Ting et al., Phys. Plasmas 4, 1889 (1997)

R.M.G.M. Trines et al., Nature Phys. 7, 87-92 (2011)

P.S. Verma, S. Sengupta, P.K. Kaw, Phys. Rev. Lett. 108, 125005 (2012)

F. Wagner et al., High Power Laser Sci. Eng. 2, e45 (2014)

H.Y. Wang et al., Phys. Rev. Lett. 107, 265002 (2011)

W.-M. Wang et al., Phys. Rev. E 91, 013101 (2015a)

W.-M. Wang et al., Phys. Rev. Lett. 114, 015001 (2015b)

W.-M. Wang et al., Phys. Rev. Lett. 114, 253901 (2015c)

W.-M. Wang et al., https://arxiv.org/pdf/1606.02437 (2016)

W.T. Wang et al., Phys. Rev. Lett. 117, 124801 (2016)

X. Wang et al., Nat. Commun. 4, 1988 (2013)

S. Weber et al., Phys. Rev. Lett. 111, 055004 (2013)

S.M. Weng et al., Optica 4, 1086-1091 (2017)

H.C. Wu et al., Phys. Plasmas 12, 113103 (2005a)

H.C. Wu et al., Appl. Phys. Lett. 87, 201502 (2005b)

X. Xie et al., Phys. Plasmas 23, 112701 (2016)

Y. Xu et al., AIP Adv. 6, 105304 (2016)

Xu Yi et al., Opt. Laser Tech. 79, 141-145 (2016)

D. Yang et al., Rev. Sci. Instrum. 85, 033504 (2014)

A. Yogo et al., Sci. Rep. 7, 42451 (2017)

J.J. Yoh et al., J. Appl. Phys. 103, 113507 (2008)

C. Yu et al., Sci. Rep. 6, 29518 (2016a)

L. Yu et al., Opt. Lett. 40, 3412 (2015)

L.L. Yu et al., J. Opt. Soc. Am. B 26, 2095 (2009)

L.L. Yu et al., Nat. Commun. 7, 11893 (2016b)

D.W. Yuan et al., Astrophys. J. 815, 46 (2015)

G. Vieux et al., New J. Phys. 13, 063042 (2011)

T. Vinci et al., Astrophys. Space. Science 298, 333 (2005)

M. Zeng et al., Phys. Plasmas 21, 030701 (2014)

M. Zeng et al., Phys. Rev. Lett. 114, 084801 (2015)

C.J. Zhang et al., Phys. Rev. Lett. 119, 064801 (2017)

H. Zhang et al., Phys. Plasmas 21, 112709 (2014)

H. Zhang et al., Plasmas 23, 082708 (2016a)

J.Y. Zhang et al., Phys. Plasmas 19, 113302 (2012)

X. Zhang et al., Phys. Rev. ST Accel. Beams 19, 101004 (2016b)

J.R. Zhao et al., Sci. Rep. 6, 27363 (2016)

Y. Zhao et al., Phys. Rev. Lett. 111, 155003 (2013)

Y. Zhao et al., Eur. Phys. Lett. 117, 65001 (2017)

C.Y. Zheng et al., Phys. Plasmas 12, 044505 (2005)

W. Zheng et al., High Power Laser Sci. Eng. 4, e21 (2016)

W. Zheng et al., Matter Radiat. Extremes 2, 243 (2017)

J.Y. Zhong et al., Astrophys. J. Suppl. 225, 30 (2016)

B.J. Zhu et al., Appl. Phys. Lett. 107, 261903 (2015) 